\title{
Gestantes, uso de plantas medicinais e educação interprofissional: relato de experiência
}

\author{
Debora Luana Ribeiro Pessoa a, Andresa Alves de Lima Galvãoa, Bruna Tamyrys da Silva \\ Fernandes ${ }^{a}$, Tays Campos Ribeiro ${ }^{a}$, Matheus Costa Cantanhede ${ }^{a}$, Henrique Morais Chagas ${ }^{a}$, \\ Micaelle das Graças Castro Bitencourt ${ }^{a}$, Jeânia Patricia dos Santos Ferreirab ${ }^{b}$, Sara Fiterman \\ Lima ${ }^{a}$, Mayara Soares Cunha Carvalho ${ }^{a}$
}

\author{
aUniversidade Federal do Maranhão - UFMA, Campus Pinheiro, Maranhão, Pará, Brasil. \\ bPrefeitura Municipal de Pinheiro, Maranhão, Pará, Brasil.
}

RESUMO O período gestacional traz a mulher uma série de modificações hormonais, podendo acarretar muitos desconfortos, como físicos e gastrointestinais. Deste modo, o presente estudo justifica-se na necessidade de levar informação sobre o uso de fitoterápicos e plantas medicinais durante a gestação. 0 estudo tem caráter qualitativo, sendo um estudo de relato de experiência, de atividade realizada durante o primeiro semestre de 2021, com o intuito de atender e orientar o público gestante da cidade de Pinheiro - Maranhão, Brasil, sobre o uso de plantas medicinais e fitoterápicos. Enquanto participantes deste processo, como docentes, discentes e preceptores, percebeu-se a importância desta atividade, pois a realização de cada etapa permitiu a absorção de conhecimentos teóricos que com certeza repercutirão na prática profissional e demandou o uso de ferramentas como trabalho em equipe, interprofissionalidade e divisão de tarefas. A fitoterapia, por ser um recurso de grande adesão popular e possuir inúmeros benefícios para prevenir e tratar diferentes enfermidades, deve ter seu uso incentivado. Contudo, é necessário que seu consumo seja feito de forma adequada e consciente para reduzir possíveis riscos e potencializar seus benefícios.

PALAVRAS-CHAVE: educação interpofissional; gestação; plantas medicinais.

Aceito 15 de maio de $2021 \quad$ Publicado online 27 de julho de 2021

Cite este artigo: Pessoa et al. (2021) Gestantes, uso de plantas medicinais e educação interprofissional: relato de experiência. Multidisciplinary Science Journal 3: e2021012, doi:10.29327/multiscience.2021012.

\section{Pregnant women, use of medicinal plants and interprofissional education: experience report}

ABSTRACT The gestational period brings to the woman a series of hormonal changes, which can cause many discomforts, such as physical and gastrointestinal. Thus, the present study is justified in need to bring information about the use of herbal medicines and medicinal plants during pregnancy. The study has a qualitative character, being a study of experience report of an activity carried out during the first semester of 2021, with the intention of attending and guiding the pregnant public in the city of Pinheiro, Maranhão, Brazil, on the use of medicinal plants and herbal medicines. As participants in this process, as teachers, students, and preceptors, the importance of this activity was perceived, as the completion of each step allowed the apprehension of theoretical knowledge that will certainly have repercussions in professional practice and demanded the use of tools such as teamwork, interprofessionality and division of tasks. Phytotherapy, as it is a widely popular resource and has numerous benefits to prevent and treat different diseases, should be encouraged to be used. Therefore, it is necessary that its consumption is done in an appropriate and conscious way to reduce possible risks and enhance its benefits.

KEYWORDS: interprofessional education; pregnancy; medicinal plants. 


\section{Introdução}

Os fitoterápicos e plantas medicinais compreendem um conjunto de espécies vegetais, cultivadas ou não, que quando administradas, exercem função terapêutica (Brasil 2019). Atualmente, essa é uma prática disseminada mundialmente e encorajada pela Organização Mundial da Saúde (OMS), especialmente em países em desenvolvimento (Mattos et al 2018).

No Brasil, o Ministério da Saúde lançou em 2006 a Política Nacional de Práticas Integrativas e Complementares (PNPIC), oferecendo aos usuários do Sistema Único de Saúde (SUS), principalmente no âmbito da Atenção Primária à Saúde (APS), inicialmente 5 Práticas Integrativas e Complementares (PICS), entre elas, a Fitoterapia. Com isso, as PICS tornaram-se um caminho para o alcance da integralidade dos usuários e o desenvolvimento da interprofissionalidade entre os diferentes profissionais das equipes de ESF e NASF (Mattos et al 2018; Previato e Baldissera 2018).

Apesar dos fitoterápicos e plantas medicinais serem introduzidos no Sistema Único de Saúde (SUS) somente no ano de 2006, seu uso faz parte da história da civilização e se dá desde os primórdios da humanidade (Mattos et al 2018; Ferreira et al 2019). O emprego dos fitoterápicos como recurso para o tratamento de enfermidades, teve seu início com base no conhecimento intuitivo, e somente com o passar do tempo aprendeu-se a diferenciar as plantas tóxicas das benéficas (Ferreira et al 2019). Hoje, o emprego dos fitoterápicos e plantas medicinais no SUS se dá a partir de evidências científicas e conforme a Relação Nacional de Plantas Medicinais de Interesse do SUS (RENISUS) publicada em 2008, contendo 71 espécies (Brasil 2019).

Vale ressaltar que após a criação da Política Nacional de Práticas Integrativas e Complementares (PNPIC), foram criados a Política Nacional de Plantas Medicinais e Fitoterápicos e o Programa Nacional de Plantas Medicinais e Fitoterápicos a fim de garantir à população brasileira o acesso seguro e o uso racional de plantas medicinais e fitoterápicos, promovendo o uso sustentável da biodiversidade, o desenvolvimento da cadeia produtiva da indústria nacional, e a elaboração de estratégias que visam atingir esses objetivos (Brasil 2006; Brasil 2015). Concomitante a isso, é vista como necessária a articulação de diferentes profissionais com ampliação do núcleo de saberes que se estendam para além do conhecimento técnico e avance em direção ao trabalho conjunto, de forma interprofissional (Previato e Baldissera 2018).

Nas últimas décadas, ocorreram muitos avanços com a formulação e implementação de políticas públicas, programas e legislação voltadas para a valorização das plantas medicinais nos cuidados primários com a saúde e sua inserção na rede pública (Brasil 2012). Além disso, apesar de já fazerem parte da cultura, houve um aumento do interesse popular por fitoterápicos, principalmente em países em desenvolvimento, onde grande parte da população é de baixa renda, graças a comprovação da sua eficácia (Ferreira et al 2019). No entanto, esse aumento da popularidade da fitoterapia nos diferentes estratos da população mundial é, em muitos casos, encorajado pela crença popular de que as preparações fitoterapêuticas são de origem natural e, portanto, são mais seguras e menos tóxicas. Isto pode levar ao uso despreocupado e desorientado por muitos indivíduos, desses medicamentos, incluindo gestantes (Cardoso e Amaral 2019).

Nesta perspectiva, é sabido que o período gestacional traz à mulher uma série de modificações hormonais, podendo acarretar muitos desconfortos, sejam físicos ou gastrointestinais. Assim, as gestantes buscam meios alternativos para cessar incômodos advindos da gestação. Todavia, são crescentes as preocupações acerca da qualidade, segurança e eficácia das preparações terapêuticas obtidas artesanalmente a partir de plantas medicinais ou de drogas vegetais cujos perfis fitoquímico, farmacológico e toxicológico não estão, em muitos casos, claramente definidos (Cardoso e Amaral 2019). Tendo e vista estes fatores, o uso indiscriminado de fitoterápicos em gestantes pode ocasionar malefícios tanto nesta quanto no feto, por efeitos teratogênicos, embriotóxicos e abortivos, sendo necessária orientação e acompanhamento médico (Jordan, Cunningham e Marles 2010).

Deste modo, o presente estudo justifica-se pela necessidade de levar informação sobre o uso de fitoterápicos e plantas medicinais durante a gestação, a fim de sugerir quais plantas devem ser evitadas e quais possuem menos riscos durante a gravidez, além de orientar sobre a forma de consumi-las e sobre a importância do diálogo com a equipe de saúde antes do uso indiscriminado desse recurso. Este estudo teve como objetivo esclarecer os frequentadores de unidades básicas de saúde, na cidade de Pinheiro - Maranhão, Brasil, em especial as gestantes, sobre o uso de plantas medicinais e fitoterápicos.

\section{Material e Métodos}


A atividade foi realizada pelo grupo tutorial de Práticas Integrativas e Complementares (PICS) do PET Saúde, da Universidade Federal do Maranhão (UFMA), Campus-Pinheiro, nos meses de janeiro, fevereiro e março de 2021, com o intuito de atender e orientar o público gestante da cidade de Pinheiro, Maranhão.

O estudo tem caráter qualitativo, do tipo relato de experiência. Para levar a informação sobre o uso de fitoterápicos e plantas medicinais em gestantes, foi feita a confecção de uma cartilha e um banner (apresentado na Figura 1), ambos de cunho informativo visando sugerir quais plantas devem ser evitadas durante a gravidez e quais possuem menos riscos à gestante, além de orientar sobre a forma de consumi-las. Para melhor acesso pela população, o material foi levado até uma unidade básica de saúde (UBS) do município, sendo lá exposto para otimizar o compartilhamento de informações à população.

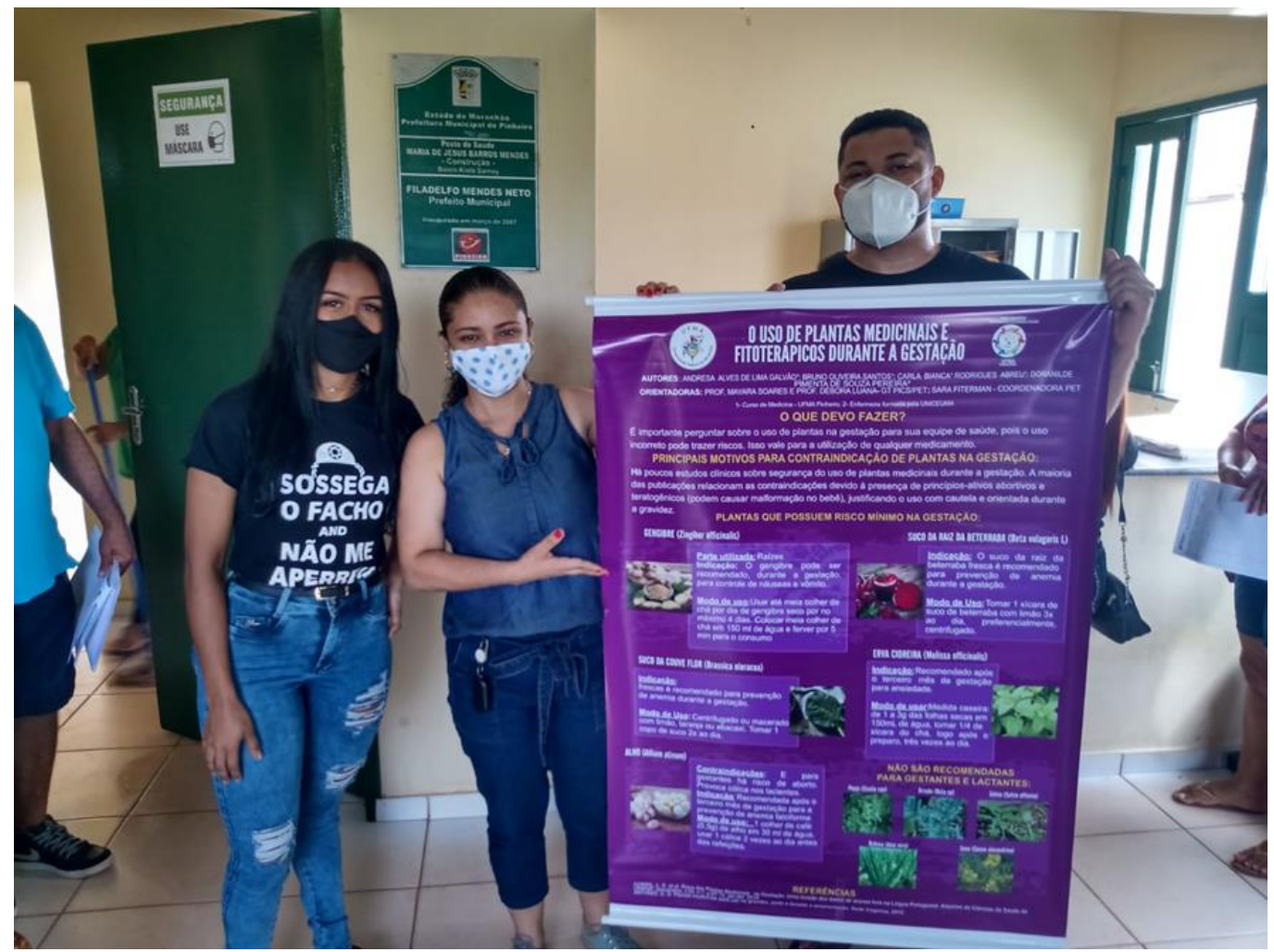

Figura 1 Registro de disponibilização do Banner sobre uso medicinal de plantas durante a gestação em Unidade Básica de Saúde do município de Pinheiro, Maranhão - 2021

\section{Resultados e discussão}

A proposta de desenvolvimento de ações que buscam democratizar o acesso ao conhecimento das práticas integrativas de maneira geral e, neste caso, especificamente dos fisioterápicos na gestação, mostram-se de altíssima importância, uma vez que essas ações contribuem para a efetivação dos princípios básicos do SUS no âmbito da atenção básica e trazem formas complementares que podem contribuir no fomento de saúde para essa população.

De acordo com Brito et al (2014) existe, de maneira geral, uma procura por plantas medicinais in natura a fim de desenvolver estratégias curativas e preventivas para variadas enfermidades e danos à saúde, tais como inflamações orofaríngeas, digestivas e respiratórias. Tal fato é bastante observado na realidade das Unidades de Saúde, onde o material produzido foi fixado. Entretanto, plantas medicinais e fitoterápicos muitas vezes são utilizados de forma inadequada ou sem nenhuma indicação de sua procedência ou cuidados, o que pode implicar em problemas diversos.

Conhecer a eficácia científica das medidas fitoterápicas contribui na aquisição de um novo modelo assistencial presente na manutenção dos sistemas orgânicos com práticas mais saudáveis e menos agressivas ao corpo, de maneira a influenciar a redução dos ganhos ou perdas em custos gerados pela utilização dos fitoterápicos (Brito et al 2014; Previato e Baldissera 2018), reforçando importância da produção de materiais informativos que ajudam não só aos pacientes, como também a equipe de assistência. 
Enquanto participantes deste processo, como docentes, discentes e preceptores percebeu-se a importância desta atividade, pois a realização de cada etapa permitiu a fixação de conhecimentos teóricos que com certeza repercutirão na prática profissional e demandou o uso de ferramentas como trabalho em equipe, interprofissionalidade e divisão de tarefas. Além disso, foi constatado a relevância de ações de extensão como a apresentada na ação descrita neste trabalho, visto que existe uma lacuna no conhecimento, devido aos poucos estudos que abordam especificamente a temática.

Esse resgate do conhecimento medicinal das plantas, tanto para os estudantes quanto para a comunidade, além de promover a integração universidade - comunidade, se apresenta como uma semente plantada com a finalidade de que esse conhecimento não se perca. Ao final de experiências como essa, é possível verificar que a utilização de plantas medicinais é muito bem aceita pela comunidade (Brito et al 2014). A Tabela 1 apresenta as espécies vegetais abordadas no material entregue às Unidades de Saúde que, de acordo com a literatura (Dante et al 2014; Izzo et al 2016; Kiguba et al 2016; Damasceno et al 2017; Nega et al 2019) apresentam risco mínimo na gestação.

Tabela 1 Espécies vegetais apresentadas na ação relacionada ao uso de plantas medicinais e fitoterápicos em gestantes, Pinheiro - Maranhão, 2021.

\begin{tabular}{|c|c|c|c|}
\hline Nome Científico & Nome Popular & Indicação & Modo de uso \\
\hline Zingiber officinalis & Gengibre & $\begin{array}{llr}\text { O gengibre } & \text { pode } & \text { ser } \\
\text { recomendado, } & \text { durante } & \text { a } \\
\text { gestação, para } & \text { controle } & \text { de } \\
\text { náuseas e vômito. } & & \end{array}$ & $\begin{array}{l}\text { Usar até } 1 \mathrm{~g} / \mathrm{dia} \quad \text { de } \\
\text { gengibre seco por no máximo } 4 \text { dias. } \\
\text { Infusão: tomar } 150 \mathrm{~mL} \text { do decocto }(0,5-5 \\
1 \mathrm{~g} \text { do rizoma seco em } 150 \mathrm{~mL} \text { de água), } 5 \\
\text { minutos após o preparo. }\end{array}$ \\
\hline Beta vulgaris $L$ & Beterraba & $\begin{array}{l}\text { O suco da raiz da } \\
\text { beterraba fresca é recomendado } \\
\text { para prevenção de anemia } \\
\text { durante a gestação. }\end{array}$ & $\begin{array}{l}\text { Tomar } 1 \text { xícara de suco de beterraba com } \\
\text { limão } 3 x \text { ao dia, preferencialmente, } \\
\text { centrifugado. }\end{array}$ \\
\hline Brassica oleracea & Couve & $\begin{array}{l}\text { O suco das folhas de couve } \\
\text { frescas é recomendado para } \\
\text { prevenção } \\
\text { de anemia durante a gestação. }\end{array}$ & $\begin{array}{l}\text { Centrifugado ou macerado } \\
\text { com limão, laranja ou abacaxi. Tomar } 1 \\
\text { copo de suco } 2 x \text { ao dia. }\end{array}$ \\
\hline Melissa officinalis & Erva cidreira & $\begin{array}{l}\text { Recomendado } \\
\text { o terceiro mês da gestação } \\
\text { para ansiedade. }\end{array}$ & $\begin{array}{l}\text { Infusão: preparar o Infuso com } 1 \text { a } 3 \mathrm{~g} \text { das } \\
\text { folhas secas em } 150 \mathrm{~mL} \text { de água, tomar } 35 \\
\mathrm{~mL} \text { do infuso, logo após o preparo, três } \\
\text { vezes ao dia. }\end{array}$ \\
\hline Allium sativum & Alho & $\begin{array}{l}\text { Recomendada após } 0 \\
\text { terceiro mês de gestação para a } \\
\text { prevenção de anemia falciforme. }\end{array}$ & $\begin{array}{l}\text { Uma colher de café } \\
(0,5 \mathrm{~g}) \text { de alho em } 30 \mathrm{ml} \text { de água, usar } 1 \\
\text { cálice } 2 \text { vezes ao dia antes das refeições. } \\
\text { Há } \quad \text { suplementos à } \\
\text { base de alho - o óleo de alho. }\end{array}$ \\
\hline
\end{tabular}

Fontes: Adaptado de Dante et al (2014), Izzo et al (2016), Kiguba et al (2016), Damasceno et al (2017) e Nega et al (2019).

Vale ressaltar que essa atividade trouxe uma experiência interprofissional importante, uma vez que se deu num processo de trabalho mediado pelo respeito, onde estudantes de cursos diferentes trabalharam juntos, num processo de compartilhamento mútuo de informações e experiências. Isto resultou numa ampliação dos métodos de ver e interpretar os fenômenos e o processo de trabalho, a partir do fomento de uma integração de saberes que culminou em uma produção que trouxe e trará benefícios para a comunidade assistida por aquela unidade de saúde. As ações interprofissionais, neste caso, reforçam a necessidade de orientação especializada no que se refere ao uso de plantas medicinais por gestantes, destacando os riscos de efeitos colaterais que o uso irracional destes produtos naturais pode ocasionar.

Dessa forma, todos os autores envolvidos foram beneficiados (alunos, professores e preceptores) pela troca de conhecimentos, pesquisa e desenvolvimento de habilidades, e a população que recebeu promoção em saúde e conhecimento direcionado, além de assistência com muito mais qualidade em um processo de troca entre a universidade e a comunidade.

\section{Considerações finais}

Este estudo objetivou esclarecer os frequentadores da unidade básica de saúde, em especial as gestantes, sobre o uso de fitoterápicos. Desse modo, o banner e a cartilha especificaram a forma de uso de cada planta medicinal, a parte 
a ser utilizada e o modo de preparo dela, a fim de maximizar o entendimento do público - alvo e tornar esse conhecimento permanente.

O aumento do uso das plantas medicinais e fitoterápicos nos diferentes grupos populacionais é associado à prática popular que, por serem de origem natural são mais seguras e menos tóxicas. Porém, vale destacar que, é preciso considerar a qualidade, segurança e eficácia das preparações terapêuticas obtidas artesanalmente a partir de plantas medicinais ou de drogas vegetais cujos perfis fitoquímico, farmacológico e toxicológico não estão, muitas vezes, claramente definidos. Sendo assim, o uso de produtos naturais pode oferecer risco à saúde de alguns indivíduos, em especial, as crianças, os idosos e as gestantes. No caso das gestantes, esse risco pode se tornar ainda maior quando há o uso concomitante de medicamentos industrializados e de preparações artesanais obtidas de plantas medicinais ou drogas vegetais, o que pode levar ao surgimento de interações medicamentosas desconhecidas na mãe e o risco de prejuízos ao feto. Dessa forma, o uso de plantas medicinais e fitoterápicos durante a gestação deve ser acompanhada por profissionais da área de saúde e pelas autoridades sanitárias, uma vez que representa uma questão relevante de saúde pública que pode envolver diferentes níveis de risco ao desenvolvimento saudável do bebê (Cardoso e Amaral 2019).

Além disso, enfatizou-se a importância de orientação e acompanhamento profissional, pois apesar de a maioria da população acreditar que plantas não possuem efeitos adversos, é sabido que há algumas contraindicações, sobretudo para gestantes e que pode haver consequências danosas em caso de uso inadequado.

Deve-se considerar, ainda, o enriquecimento de conhecimento e experiência para a equipe de práticas integrativas do PET saúde, que além de levar informações a sociedade, também pode colocar em prática princípios de interprofissionalidade e colaboração com execução da atividade.

Conclui-se que a fitoterapia por ser um recurso de grande adesão popular e possuir inúmeros benefícios para prevenir e tratar diferentes enfermidades, deve ter seu uso incentivado. Para tanto, é necessário que seu consumo seja feito de forma adequada e consciente para reduzir possíveis riscos e potencializar seus benefícios.

\section{Declaração de conflito de interesses}

Os autores declaram que não há conflitos de interesse. Todos os autores estão cientes da submissão do artigo.

\section{Contribuição dos autores}

PESSOA, Débora Luana Ribeiro Pessoa; GALVÃO, Andresa Alves de Lima; FERNANDES, Bruna Tamyrys da Silva; RIBEIRO, Tays Campos; CANTANHEDE, Matheus Costa - Redação, revisão e formatação do artigo.

CHAGAS, Henrique Morais; BITENCOURT, Micaelle das Graças Castro; FERREIRA, Jeânia Patricia dos Santos; CARVALHO, Mayara Soares Cunha - Revisão do artigo.

\section{Referências}

Brasil (2019) Práticas Integrativas e Complementares (PICS): quais são e para que servem. Ministério Da Saúde. Disponível em: https://antigo.saude.gov.br/saude-de-a-z/praticas-integrativas-e-complementares. Acesso em: 29 de setembro de 2021.

Brasil. Ministério da Saúde (2006) Política Nacional de Plantas Medicinais e Fitoterápicos (Série B. Textos Básicos de Saúde). Disponível em: https://bvsms.saude.gov.br/bvs/publicacoes/politica nacional fitoterapicos.pdf. Acesso em: 29 de setembro de 2021.

Brasil. Ministério da Saúde (2015) Programa Nacional de Plantas Medicinais e Fitoterápicos. Disponível em: http://bvsms.saude.gov.br/bvs/publicacoes/programa nacional plantas medicinais fitoterapico 1ed.pdf. Acesso em: 29 de setembro de 2021.

Brasil. Ministério da Saúde (2012) Práticas Integrativas e Complementares: Plantas Medicinais e Fitoterapia na Atenção Básica. (Série A. Normas e Manuais Técnicos). Disponível em: http://bvsms.saude.gov.br/bvs/publicacoes/praticas integrativas complementares plantas medicinais cab31.pdf. Acesso em: 29 de setembro de 2021. 
Brito AGR, Freitas CL, Galvão RC, Nunes JT, Silva JL, Emiliano MDS, Santos RS (2014) Fitoterapia: uma Alternativa Terapêutica para o Cuidado em Enfermagem - Relato de Experiência. Biota Amazônia. DOI: 10.18561/2179-5746/biotaamazonia.v4n4p15-20

Cardoso BS, Amaral VCS (2019) O uso da fitoterapia durante a gestação: um panorama global. Ciência \& Saúde Coletiva. DOI: $10.1590 / 1413-81232018244.07472017$

Damasceno D, Leal-Silva T, Soares T, Moraes-Souza R, Volpato, G (2017) Medicinal Plants for Diabetes Treatment During Pregnancy. Current Medicinal Chemistry. DOI: 10.2174/0929867323666161003122914

Dante G, Bellei G, Neri I, Facchinetti F (2014) Herbal therapies in pregnancy. Current Opinion in Obstetrics \& Gynecology. DOI: $10.1097 /$ GCO.0000000000000052

Ferreira ET, Santos ES, Monteiro JS, Gomes MSM, Menezes RAO, Souza MJC (2019) The use of medicinal and phytotherapy plants: an integrational review on the nurses performancee. Brazilian Journal of Health Review. Disponível em: https://www.brazilianjournals.com/index.php/BJHR/article/view/1383/1260. Acesso em: 29 de setembro de 2021.

Izzo AA, Hoon-Kim S, Radhakrishnan R., Williamson EM (2016) A Critical Approach to Evaluating Clinical Efficacy, Adverse Events and Drug Interactions of Herbal Remedies. Phytotherapy Research. DOI: 10.1002/ptr.5591

Jordan SA, Cunningham DG, Marles RJ (2010) Assessment of herbal medicinal products: Challenges, and opportunities to increase the knowledge base for safety assessment. Toxicology and Applied Pharmacology. DOI: 10.1016/j.taap.2009.12.005.

Kiguba R, Ononge S, Karamagi C, Bird SM (2016) Herbal medicine use and linked suspected adverse drug reactions in a prospective cohort of Ugandan inpatients. BMC Complementary and Alternative Medicine. DOI: 10.1186/s12906-016-1125-x

Mattos G, Camargo A, Sousa CA, Zeni ALB (2018) Plantas medicinais e fitoterápicos na Atenção Primária em Saúde: percepção dos profissionais. Ciência \& Saúde Coletiva. DOI: 10.1590/1413-812320182311.23572016

Nega SS, Bekele HM, Meles GG, Nordeng H (2019) Medicinal Plants and Concomitant Use with Pharmaceutical Drugs Among Pregnant Women. The Journal of Alternative and Complementary Medicine. DOI: 10.1089/acm.2018.0062

Previato GF, Baldissera VDA (2018) Retratos da prática interprofissional colaborativa nas equipes da atenção primária à saúde. Revista Gaúcha de Enfermagem. DOI: 10.1590/1983-1447.2018.2017-0132 